\title{
Type II hypersensitivity and trimethoprim-sulfamethoxazole
}

\author{
Allen $\mathbf{S}^{1}$, Ervin $\mathbf{S}^{2}$ \\ ${ }^{1}$ Shelby Allen*, BS, Department of Pediatrics, Wake Forest School of Medicine, ${ }^{2}$ Sean Ervin, MD PhD, Associate \\ Professor of Medicine and Pediatrics, Wake Forest School of Medicine, Medical Center Boulevard, Winston-Salem, NC \\ 27157 \\ *Department of Surgery, Medical University of South Carolina, Charleston, South Carolina. \\ Address for Correspondence: Sean Ervin, Associate Professor of Pediatrics, Wake Forest Baptist Medical Center, \\ Medical Center Boulevard, Winston-Salem, Email: servin@wakehealth.edu
}

\begin{abstract}
Type II hypersensitivity reaction is an unusual response to trimethoprim-sulfamethoxazole.We discuss a case of rash and pancytopenia immediately following the use of trimethoprim-sulfamethoxazole in an adolescent female.
\end{abstract}

Key words: Drug eruptions, Hypersensitivity, Pancytopenia, Sulfamethoxazole

\section{Introduction}

Trimethoprim-sulfamethoxazole (Septra) is a widely used antibiotic world-wide.The clinical use has been increasing in the pediatric population[1].

Septra has been associated with a broad array of drug associated reactions including gastrointestinal complaints, cutaneous reactions including StevensJohnson syndrome and toxic epidermal necrolysisand cytopenias including immune mediated thrombocytopenia[2].

Adverse reactions occur in $6-8 \%$ of patients. In the pediatric patient hospitalized for an adverse drug reaction priorexposure to Septra is found in $75 \%$ of patients [3].In the case presented we describe a cutaneous reaction to Septra clinically consistent with a Type2 hypersensitivity reaction with associated pancytopenia. Idiosyncratic reactions such as Type 2 hypersensitivity have rarely been reported with Septra exposure.These adverse drug reactions have infrequently been reported to be fatal [4].With the increasing use of Septra for the management of community acquired methicillin resistant Staphylococcus aureus of skin and soft tissue infections [5] clinicians will need to recognize this clinical complication.

Manuscript received: $14^{\text {th }}$ Feb 2016

Reviewed: $22^{\text {nd }} \mathrm{Feb} 2016$

Author Corrected; $4^{\text {th }}$ March 2016

Accepted for Publication: $18^{\text {th }}$ March 2016

\section{Case Report}

A 16-year-old female presented with rash. Two weeks prior to presentation she had afoot abscess drained and received cephalexin.Five days latershe was switched to trimethoprim-sulfamethoxazole (Septra). Five days prior to presentationshe developed pruritic'small, red bumps' diffusely over her body.The day before presentation, she developed fever, chills, nausea, and headache.At presentation, she was febrile $\left(103^{\circ} \mathrm{F}\right)$ and had facial redness and swelling. Atthe time of presentation she had been on trimethoprimsulfamethoxazoletwice daily for 6 days and reported having missed 2-3 doses. This was her first known exposure to trimethoprim-sulfamethoxazole.The patient received a $125 \mathrm{mg}$ dose of methylprednisolone (Solumedrol)and a hemogram demonstrated pancytopenia. Shewas referred to our institution for further management.

On arrival, the hemogram showed: WBC $2,000 / \mu \mathrm{L}$ (78\% neutrophils), ANC 1,600; hemoglobin $11.5 \mathrm{~g} / \mathrm{dL}$; and platelets $115,000 / \mu \mathrm{L}$. Reticulocyte count was $0.3 \%$. Screening for a possible hemolytic anemia demonstrated the Lactate dehydrogenase was 373 U/Land uric acid was $2.7 \mathrm{mg} / \mathrm{dL}$. The patient was clinically stable.Physical examination revealed a generalized rash involving the upper and lower extremities, abdomen, chest, and back with no involvement of the palms or soles.The rash was 
characterized by petechiae most prominently on the legs (Figure 1) but also on the palate.The drained abscess was noted to have a well-healing incision site, mild erythema and swelling, but no fluctuance or expressible pus. A $5 \mathrm{~cm}$ by $5 \mathrm{~cm}$ hard, nontender nodule was palpable behind the right ear; no other lymphadenopathywas present.Based on the clinical characteristics of the rash we concluded the patient demonstrated a type II hypersensitivity reaction to trimethoprim-sulfamethoxazole.The drug was discontinued at admission.

Figure1: Characteristic rash involving lower extremities

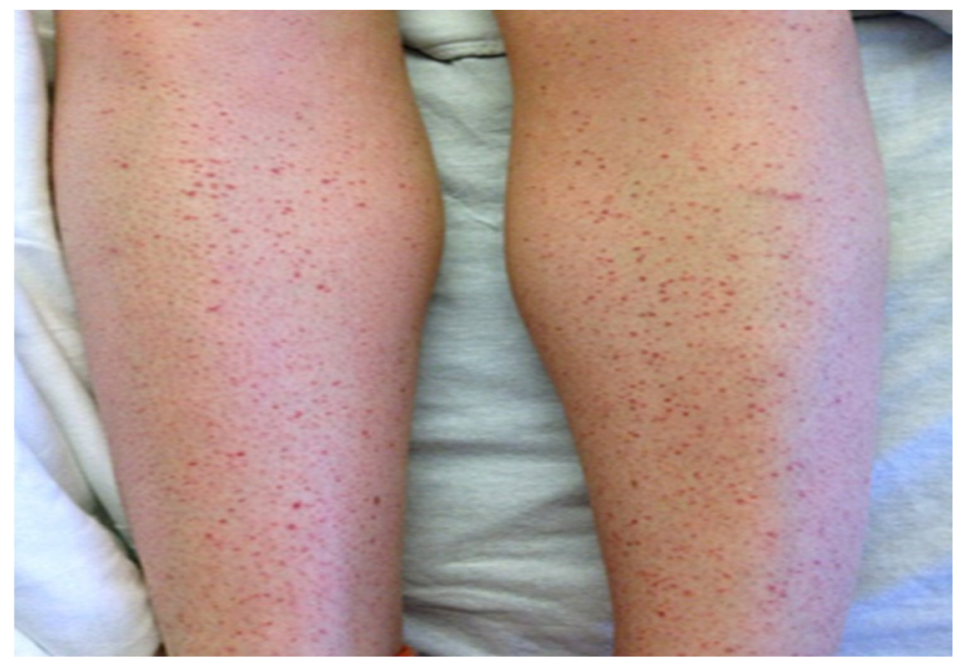

Table1: Complete blood counts at admission and three days following discharge.

\begin{tabular}{|l|l|l|}
\cline { 2 - 3 } \multicolumn{1}{c|}{} & Admission Date & Post-Discharge Day 3 \\
\hline WBC (10 $/ \boldsymbol{\mu} \mathbf{L})$ & 2.0 & 4.6 \\
\hline Hemoglobin $(\mathbf{g} / \mathbf{d L})$ & 11.5 & 12.4 \\
\hline Platelets $(\mathbf{1 0} / \boldsymbol{\mu L})$ & 115 & 186 \\
\hline
\end{tabular}

The patient remained in the hospital for two days, during which time she had modest clinical improvement.A hemogram three days after discharge indicated the patient's bone marrow to be recovering (Table 1). She was contacted 13 days after discharge and reported that the rash was resolved, she was afebrile, and she had required no further treatment.

\section{Discussion}

The patient's recent exposure to Septra, stable condition, and the characteristic pruritic maculopapular rash led us to believe that drug hypersensitivity was the most likely explanation of her symptoms. The petechial nature of the rash was concerning and not entirely consistent with the relatively minor degree of thrombocytopenia. Our diagnosis for this patient wasa type II hypersensitivity reaction, howeverother severe and potentially life- threatening diagnoses were considered, including rickettsial disease, (endemic in our region) and hematologic malignancy.As there was no eosinophilia noted we did not feel this represented a drug reaction with eosinophilia and systemic symptoms(DRESS syndrome).

The normal lactate dehydrogenase and uric acid levelsruled out a hemolytic process or tumor lysis. The reticulocyte count at $0.3 \%$ indicated bone marrow suppression. Uncommonly, neutropenia/ agranulocytosis has been associated with trimethoprimsulfamethoxazole [6].

\section{Conclusion}

Cutaneous reactions to Septra have been well described and include urticarial, maculo-papular and papular exanthems. Rarely Stephens Johnson Syndrome and 
Toxic Epidermal Necrolysis have been reported [7]. Most drug hypersensitivity reactions are types I and IV; types II and III are less common and associated with a higher doseorprolonged therapy with the offending drug. Thecase presented here demonstrated features of a type II hypersensitivity reaction.Commonhematologic manifestations of type II drug reactions are neutropenia, hemolytic anemia, and thrombocytopenia; characteristic features of this case. Symptoms usually do not appear until at least 5 to 8 days following administration of the drug, as was true in this case [8]. Idiosyncratic reactions may occur including agranulocytosis and sepsis like hypersensitivity syndrome [9] which are rarely fatal. The physician needs to be aware of the wide range of cutaneous and systemic manifestations associated with

\section{References}

1. HayashiM, StrouseJ, Veltri M, Curtis BR, Takemoto CM.Immune thrombocytopenia due to TrimethoprimSulfamethoxazole; under-recognized adverse drug reaction in children?Pediatr Blood Cancer. 2015; 62(5):922-923.doi: 10.1002/pbc. 25430

2. Aster RH, Bougie DW. Drug-induced immune thrombocytopenia. N Engl J Med. 2007; 357(6):580 587.

3. Goldman J, Jackson MA, Herigon JC, Hersh AL, Shapiro DJ, Leeder JS. Trends in Adverse Reactions to Trimethoprim-Sulfamethoxazole.Pediatrics. 2013; 131(1): e103-e108.doi: 10.1542/peds.2012-1619

4. Kocak Z, Hatipoglu CA, Ertem G, Kinikli S, Tufan A, Irmak $\mathrm{H}$, et al.. Trimethoprim-sulfamethoxazole induced rash and fatal hematologic disorders. Jnfect. 2006; 52(2):e49-52. exposure to Septra; while these are rarely fatal, the extent and characteristics of the drug reaction may represent a poor outcome [4]. Close follow-up with the patient including serial blood counts is important to ensure normalization of the blood counts. In this case with the discontinuation of the drug the patient returned to her normal state of health with normalization of the hematologic parameters.

\section{Conflict of Interest:}

Shelby Allen reports no conflict of interest Sean Ervin reports no conflict of interest.

Funding: No Funding

Conflict of Interest: The authors declare no conflict of Intere.

5. Lee MC, Rios AM, Aten MF, Mejias A, Cavuoti D, McCracken GH, Jr, et al. Management and outcome of children with skin and soft tissue abscesses caused by community-acquired methicillin-resistant Staphylococcus aureus. Pediatr InfectDis J. 2004; 23(2):123-127.

6.Curtis BR. Drug-induced immune neutropenia/agranulocytosis.Immunohematology. 2014; 30(2):95-101.

7. SchopfE. Skin reactions to co-trimoxazole. Infection 1987; 15 Suppl 5: S254-258.

8. Pichler WJ. Drug allergy: classification and clinical features. In: UpToDate,Feldweg AM (Ed), Waltham, MA, 2013.http://www.uptodate.com.(Accessed on April 26, 2014.)

9.MainraRR, Card $\quad$ SE.Trimethoprimsulfamethoxazole-associated hepatotoxicity-part of a hypersensitivity syndrome. Canadian Journal of Clinical Pharmacology.2003; 10 (4):175-178

\section{How to cite this article?}

Allen S, Ervin S, Type II hypersensitivity and trimethoprim-sulfamethoxazole. Pediatr rev. Int J Pediatr Re 2016;3(3):199-201.doi:10.17511/ijpr.2016.i03.11. 